\title{
Synthesis of Spiro-[Butyrolactone- Pyrrolidine]
}

\section{Category}

Metal-Catalyzed

Asymmetric

Synthesis and

Stereoselective

Reactions

\section{Key words}

azomethine ylides

copper

spirocycles
Significance: The authors developed an asymmetric synthesis of spiro-[butyrolactone-pyrrolidine] catalyzed by $\mathrm{Cu}(\mathrm{I})$-DTBM-BIPHEP delivering exo-selective 1,3-dipolar cycloadducts of azomethine ylides and $\alpha$-methylene- $\gamma$-butyrolactone. In all cases excellent chemical yields and stereoselectivities were achieved.
Comment: Several natural alkaloids and important biological compounds contain spiro-[butyrolactone-pyrrolidine] as core structure making them very attractive targets in the synthetic community. Thus, this finding for the syntheses of bicyclic and tricyclic skeletons with multiple quaternary stereogenic centers is very attractive. 